\title{
Using PDES to Simulate Individual-Oriented Models in Ecology: A Case Study
}

\author{
Remo Suppi, Pere Munt, and Emilio Luque \\ Dept. of Computer Science, University Autonoma of Barcelona, \\ 08193, Bellaterra, Spain \\ Remo.Suppi@uab.es Pere.Munt@campus.uab.es Emilio.Luque@uab.es
}

\begin{abstract}
The present work outlines the results of the Parallel Discrete Event Simulation (PDES) utilization for solving an individual based model: Fish Schools. This type of model cannot be solved through analytical methods, thus simulation techniques are necessary. The greater problem presented by this type of model is the high computing capacity necessary for solving middle-high size problems (hundreds to thousands of individuals). PDES is presented as a useful and low cost tool for individual-oriented models simulation, since it allows a scalable and efficient solution in accordance with the problem to be simulated.
\end{abstract}

\section{Introduction}

One of the main drawbacks to the use in ecology of individual-oriented models is the necessary computing capacity for their simulation. This type of model chooses the individual as basic element of the system. The ecosystem is described by dynamic and static individuals properties. The behaviour of an individual can differ from the behaviour of other individuals of the same or other species. This type of model cannot be solved in analytical form and it is therefore necessary to use simulation techniques in obtaining the ecosystem's dynamical behaviour. For complex systems (thousands of individuals), it is necessary to use advanced simulation techniques and paralleldistributed computing systems to give an efficient response to this type of problems. PDES (parallel and distributed event simulation) is a useful tool (and indispensable in some instances) for providing response to complex problems within an acceptable time. There are two main objectives to PDES: to reduce the simulation execution time, and to increase the potential dimension of the problem to be simulated. This paper demonstrates the use of PDES in solving a type of individual-oriented model: Fish Schools. The next section is a summary of the individual-orient model characteristics. Section 3 shows the implementation of the model on a distributed simulator. Section 4 presents the experimental framework and sections 5 and 6 the conclusions and references, respectively.

${ }^{1}$ This work has been supported by the CICYT under contract TIC98-0433 


\section{Individual-oriented models}

There are considerable references to individual-oriented models (IoM) in the literature $[3,7,6]$. The model definition is based on recognizing each individual as an autonomous agent that acts according to a set of biological rules.

One of the most representative applications of IoM is used to describe the movement of given species (schools, flocks, herds, etc) [5,6]. The IoM utilization allows us to determine the movement of a species group by using the movement of each member. The Fish Schools is an IoM application for the movement of fish species. $[8,6,10]$. From the observation, was discovered that fish can describe very complex figures in their movement, but that these figures are governed by three basic postulates from the point of view of the individual:

- To avoid collisions

- Speed coupling

- To obtain a position in the centre of the group.

These rules express both the individual's need for survival and its instinct for protection (the need to escape from predators). Each fish in the model is represented as a point in a three-dimensional space with an associated speed. And each fish changes position and speed simultaneously after a certain period $\Delta \mathrm{t}$. The actions that the model describes for each fish are:

1- $\quad$ Each fish chooses up to $X$ neighbour fish $(X=4$ seems sufficient for most schools), which will be those nearest and with direct vision.

2- Each fish reacts in accordance with the direction and distance of each neighbour. Three influence radios and three possible reactions are established. The final reaction will be the average of the reactions experimented on each neighbour.

a. If the neighbour is found within the smaller radio, the fish will carry out an "opposed to address" movement -repulsion action- (to avoid collisions).

b. If the neighbour is within a second influence radio, the fish will adopt the same direction as the neighbour.

c. If the neighbour is within a third radio, the fish will move towards it.

3- Each fish calculates its new position according to the new direction.

This generates a very simple model, but one that allows very complex behaviour to be described (an implementation with applets in 2D can be found in [4]). As a counterpart, very high computing power is necessary, since the complexity algorithm is of $\mathrm{O}\left(\mathrm{N}^{2}\right)$, where $\mathrm{N}$ is the number of fish (each fish attempts to find the neighbour fish by inspecting all other fish in the school).

Each fish Fi is defined by its position $\overrightarrow{p_{i}}$ and velocity $\overrightarrow{v_{i}}$, and chooses its potential neighbours by watching concentric zones of incremental radio until finding $\mathrm{X}$ fish. To calculate the distance between two fish, the Euclidean distance is used: 


$$
\operatorname{Dist}\left(p_{a,} p_{b}\right)=\sqrt{\left(p_{a x}-p_{b x}\right)^{2}+\left(p_{a y}-p_{b y}\right)^{2}+\left(p_{a z}-p_{b z}\right)^{2}}
$$

The potential neighbours are chosen by using the algorithm of front priority. This algorithm calculates all the angles formed by $\overrightarrow{v_{i}}$ and $\overrightarrow{p_{i}}-\overrightarrow{p_{j}}$ (being the collision angle between $p_{i}$ and $p_{j}$ ); the $X$ neighbours with the smallest angle are chosen. Figure 1 shows the neighbour selection of $p_{1}$ using the front priority algorithm. $p_{2}$ is the neighbour selected, since the angle $v_{1}-v_{21}\left(v_{2},\left(p_{2}-p_{1}\right)\right)$ is less than $v_{1}-v_{31}\left(v_{3},\left(p_{3}-p_{1}\right)\right)$.

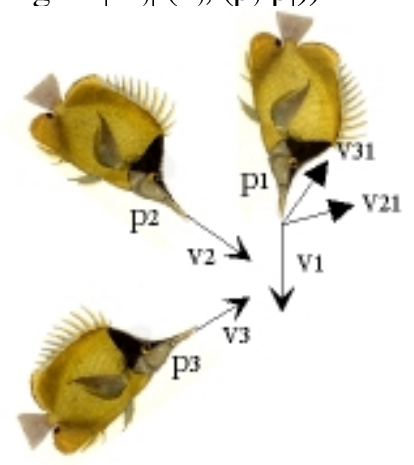

Fig. 1. Neighbour selection

Each $\mathrm{F}_{\mathrm{i}}$, once they have selected the $\mathrm{X}$ neighbours, must then determine the reaction (rotation of the $\mathrm{v}_{\mathrm{i}}$ ) to each $\mathrm{F}_{\mathrm{j}} \beta_{i j}$ will be the $\mathrm{F}_{\mathrm{i}}$ reaction with respect to $\mathrm{F}_{\mathrm{j}}$ expressed in spherical coordinates. Each fish $\mathrm{F}_{\mathrm{j}}$ can be found within one of three possible areas of influence with respect to $\mathrm{F}_{\mathrm{i}}$ (A: near, B: middle, $\mathrm{C}$ : far):

- If Dist $\left(F_{j}, F_{i}\right) \leq A, F_{i}$ has a repulsion reaction with respect to $F_{j}$ and $\beta_{i j}=(1, \pi, \pi)$

- If $A<\operatorname{Dist}\left(\mathrm{F}_{\mathrm{j}}, \mathrm{F}_{\mathrm{i}}\right) \leq \mathrm{B}, \mathrm{F}_{\mathrm{i}}$ adopts a parallel position with respect to $\mathrm{F}_{\mathrm{j}}$ and $\beta_{\mathrm{ij}}=\left(1, \theta_{\mathrm{j}}, \varphi_{\mathrm{j}}\right)$

- If $B<\operatorname{Dist}\left(\mathrm{F}_{\mathrm{j}}, \mathrm{F}_{\mathrm{i}}\right) \leq \mathrm{C}, \mathrm{F}_{\mathrm{i}}$ is guided toward $\mathrm{F}_{\mathrm{j}}$ and $\beta_{\mathrm{ij}}=\left(1, \theta_{\mathrm{i}}-\theta_{\mathrm{j}}, \varphi_{\mathrm{i}}-\varphi_{\mathrm{j}}\right)$

Finally, reaction $\beta$ is obtained (mean value for all $\beta_{\mathrm{ij}}$ ) and $\mathrm{v}_{\mathrm{i}}$ is rotated according to $\beta$. Object-Oriented methodology and UML techniques [1] were used for the model implementation and the analysis phase, respectively. [5]

\section{PDES simulation}

As model of the parallel discrete event simulation (PDES), a set of logical processes (LP) managing a distributed event lists was considered. These processes interact exclusively by exchanging time-stamped messages. The PDES mechanisms can be divided in two categories: conservative and optimistic. Conservative approaches use synchronization to avoid causality errors. In these algorithms, the events are processed when it is certain that the execution order is correct. On the other hand, in optimistic algorithms, each LP processes the events as soon as they are available and this execution, in some instances, can mean causality errors. Nevertheless, the algorithm has detection mechanisms to avoid these errors and to recover the causality $[11,12,9]$. 
The fish school simulator was built on the base of a PDES simulation kernel developed at the UAB (written in $\mathrm{C}++$ ). This kernel is designed to be executed in Unix stations and PVM. The union of the simulation kernel and the model classes is accomplished through the inheritance of the kernel classes and virtual functions implementation. This simulation kernel uses an STL library for data structure management. $[9,2]$. Based on the UAB kernel, two simulator versions were developed: sequential and distributed. The distributed version will be used to make performance analysis using different simulation PDES algorithms. The sequential version will allow speedup and performance analysis to be undertaken with respect to the distributed version.

\subsection{Model distribution}

One of the most important problems than a model of these characteristics outlines is the individual's distribution within distributed architecture computing elements. Lorek [8] outlines a distribution of a Fish School simulator for real time visualization. The distribution is accomplished by assigning a static partition of the fish group to simulate in each processor, but each processor has the information on the fish assigned in other processors.

Our solution is based on the problem division in a set of logical processes (LP), which will be executed in the different processors. For each LP, an initial partition of the problem (number of fish) is assigned and this quantity will change dynamically during the simulation. The LPs have a physical zone of the problem (cubes in fig. 2) to simulate (Spatially Explicit Simulation) and the fish movement will imply migrations between the LPs.

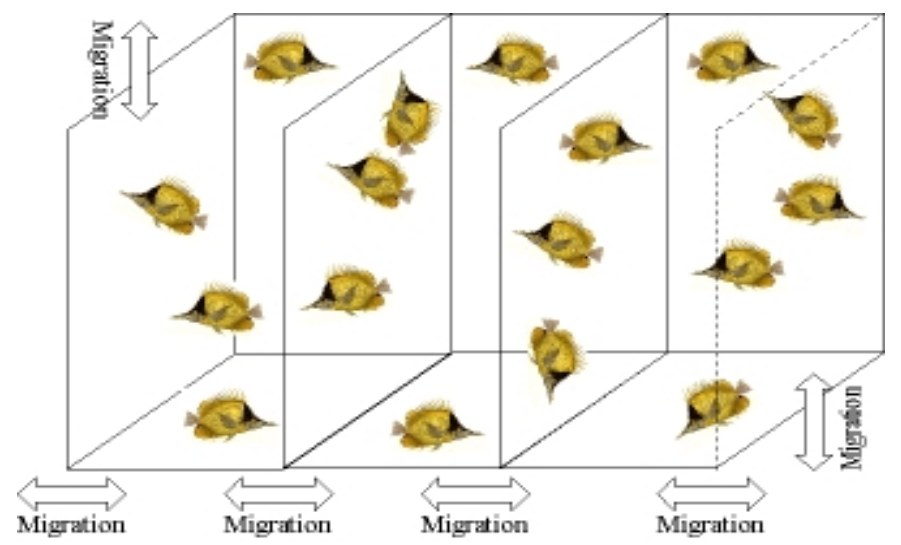

Fig. 2. Model distribution

The present model implements two types of messages between LPs: neighbour petition within a given zone and migration. To reduce the information quantity that the LPs should share, a point-to-point petition was designed (under demand): only the neighbours of a specific zone are requested. If the LP simulated area is a cube, only the neighbours that share a cube side will be requested (see fig. 2). 


\section{Experimental Studies}

The experimental framework was developed on a cluster of machines executing Linux SuSE 6.4 and interconnected by Fast Ethernet. The tools used for the development were: Pvm 3.4.3, Xpvm 1.1.5, Gcc 2.95 and Ddd 3.2 (Date Display Debugger). Figure 3 a,b,c shows three types of animation frames obtained from the simulator traces.

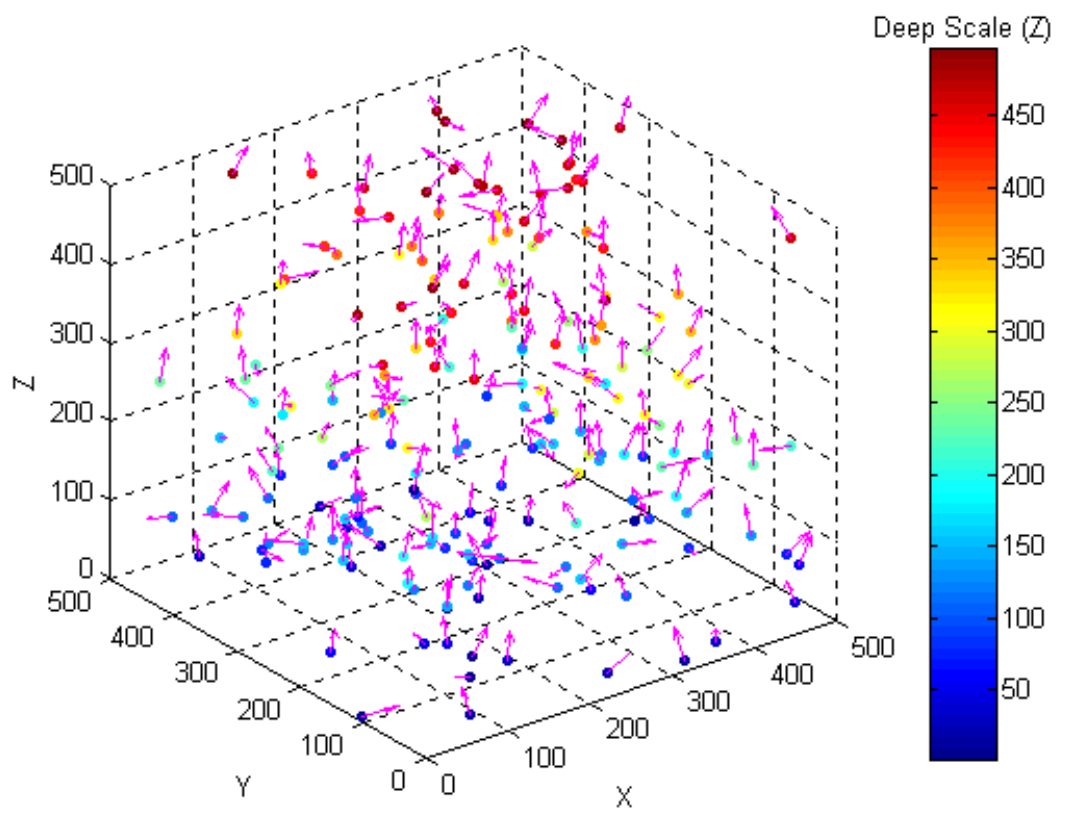

Fig. 3.a 3-D Fish Visualization
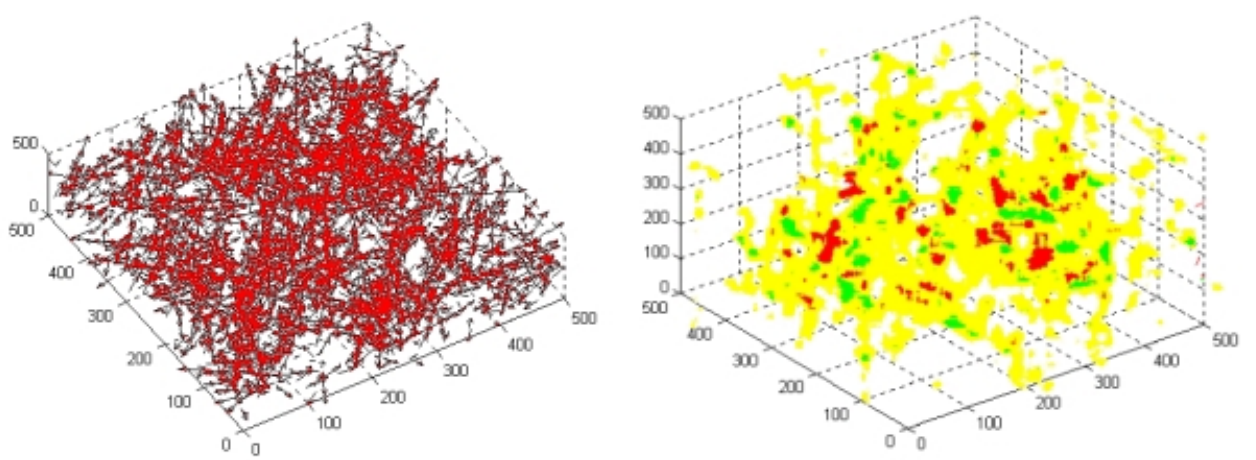

Fig. 3.b, c. Visualization of trajectory \& density of fish (10 frame animations) 
Figure 3.a shows a distribution of 300 fish. The point and colours indicate their position and depth (with respect to the colour scale), respectively, and the arrows indicate their direction and speed. Figure 3.b shows a path graph for 300 fish during a 100-frame simulation, and figure 3.c shows fish density in a given zone (red=high density, green=low density).

The first step in the development process was the design and development of the sequential simulator version. This version was analysed with different territory sizes (up to 800:100:100), number of individuals $(100,200,400,800,1600)$, constant velocity and constant neighbours number ( 5 and 4 respectively) and constant influence radios $\left(R_{1}=5, R_{2}=20, R_{3}=30\right)$ to verify the model and to validate the simulator. As reference measure, we chose a sample of the real complexity and frame generation time (new position and speed for each individual) for colonies of 100 to 1600 fish (figure 4). This graph shows that animations in real time in the sequential simulator have a complex treatment, since with groups of 200 individuals, 1 second per frame is needed.

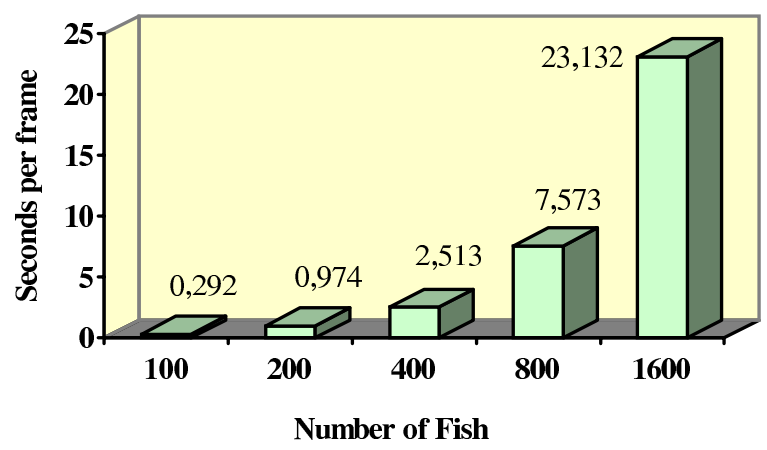

Fig. 4. Seconds per frame in the sequential simulator

After the sequential version, the following step was the development of distributed versions. The first objective was to build the simulator under an optimistic algorithm (Time Warp) and to solve the load balance between the LPs, in order to avoid synchronizations. The results under this simulation method are acceptable, but do not have a homogeneous behaviour for different fish colonies (as was expected). Under the Time Warp algorithm (optimistic), the model shows acceptable behaviour for small quantities of fish $(<100$ individual) on large territories $(800 \times 100 \times 100)$ where migration events are few and therefore the need for synchronization is minimal. With a more realistic evaluation of fish quantities ( $>100$ individual), the simulation model was unable to advance due to the great quantity of external events produced both by migration and neighbour request. The main problem with the Time Warp algorithm is the rollback chains [11]. In an IoM of these characteristics, rollback chains are produced easily in the migration and neighbours petition actions. The problem is due to the fact that the fish do not have a regular movement and often come and go on the same point (see figure 3.b). This behaviour generates a very high quantity of migra- 
tions on the LPs shared cube face. Such activity means that synchronization between the two LPs is very difficult.

The second step was the utilization of conservative distributed simulation algorithms to control the synchronization events. Our objective was to avoid the 'out of order' events processing to remove the synchronization events. Each $\mathrm{LP}_{\mathrm{j}}$ will have to execute the following steps (in each iteration):

1. To generate the iteration results $\mathrm{T}_{\mathrm{j}, \mathrm{i}}$ (state + migrations).

2. To wait until all the $\mathrm{LP}_{\mathrm{kk} \mathrm{j}_{\mathrm{j}}}$ sharing information with $L P_{j}$ finish their iteration $T_{k, i^{*}}$

3 . To process the external events.

4. To generate the iteration $\mathrm{T}_{\mathrm{j}, \mathrm{i}+1}$

The worse case is when an LP does not know with which LP shares information. In this case, a global waiting time will be necessary and therefore the global simulation time progression (GVT) will be conditioned by the speed of the slowest LP. This situation does not exist when the experiments are accomplished on homogeneous machines and similar computing charges. Figure 5 shows the time per frame for a conservative distributed simulation on 1,2,4,6,8 processors. The simulation characteristics are: territory $=800: 100: 100$; individual $=100,200,400,800,1600$; speed $=5$; neighbour number $=4$; influence radio $=5: 20: 30$.

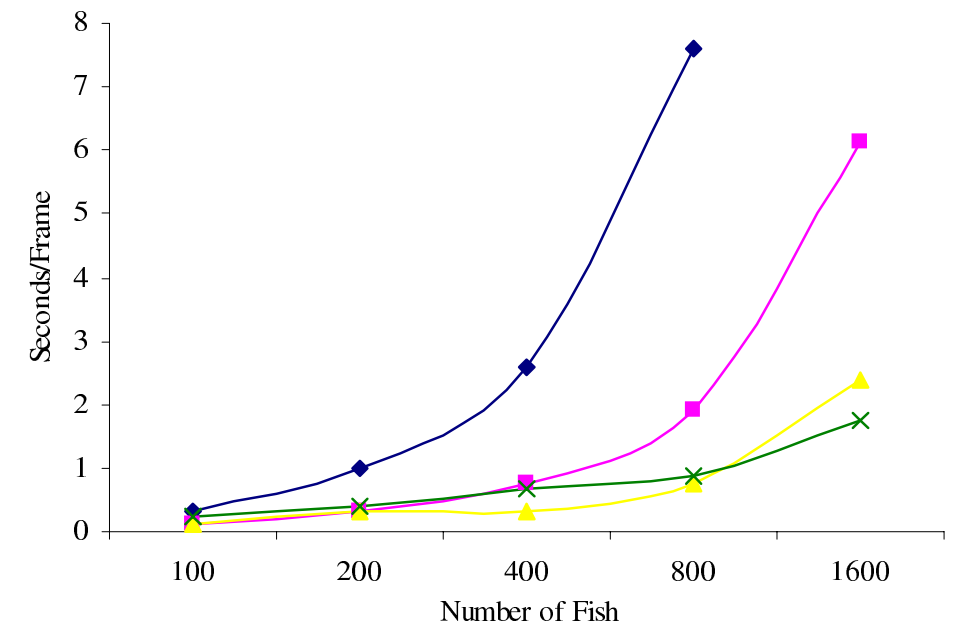

$\multimap 1$ Processor $\rightarrow-2$ Processors $\longleftarrow 4$ Processors $\multimap 8$ Processors

Fig. 5. Conservative fish school simulation

From figures 5 and 6 , the following conclusions can be extracted:

- The model scales well: as a rule, an increase in processor number facilitates a reduction in frame time to values below 1 second.

- The frame generation time cannot be reduced without the limit increasing processor number. Figure 6 shows that the tendency line for 1600 fish is asymptotically to $0,15 \mathrm{~s}$ (decreasing this time would require acting on the communications model). For visualizations in real time, approximately 4 fps for 400 fish and 4 processors are obtained. 
- The model to be simulated must be analysed carefully, there are situations in which adding processors does not bring about benefits. This case is present in the current model (fig. 4): it is not necessary to use 8 processors to simulate below 800 individuals (the cube generates a high communication granularity for these cases).

- The results obtained are excellent, but results above the linearity require explanation.

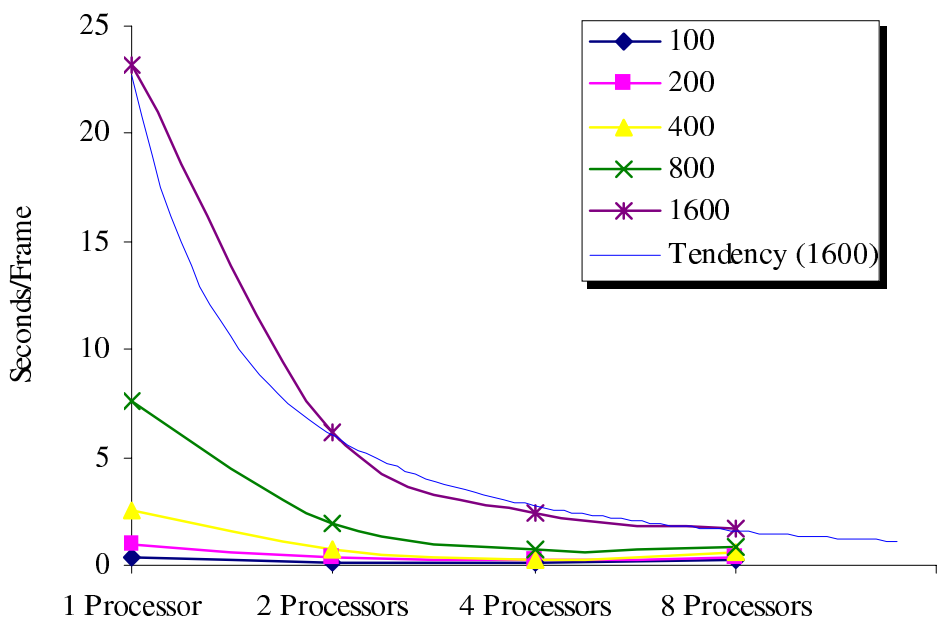

Fig. 6. Improvement \& tendency line for the simulation of 1600 individuals

Figure 7 shows speedup with respect to the sequential version and for different fish colonies. This figure confirms that at least 100 fish per processor are required in order to obtain acceptable speedups. A comment with respect to this profit is necessary. Parallel programs use linear speedup as maximum profit, but figure 7 show that the profit obtained is superior in given points. This singularity is explained by the model complexity calculation. The IoM Fish Schools has complexity $\mathrm{O}\left(\mathrm{N}^{2}\right)$ [8], since the data structure that sustains the individuals does not have the implicit location (neighbours are found searching in $\mathrm{N}$ fish positions). The partition generation (section 3.1) adds this information implicitly: each process must accomplish the neighbour's searches in the current partition and, eventually, in the shared cube faces. In this way, the complexity for two processes is $\mathrm{O}\left(2 *(\mathrm{~N} / 2)^{2}\right)$, and $2 *(\mathrm{~N} / 2)^{2}$ is less than $\mathrm{N}^{2}$. In this sense, two comments are required:

a) The model distribution generates a considerable additional advantage that must be taken into account in the PDES speedup calculation.

b) The model series was not implemented with complexity $\mathrm{O}\left(\mathrm{k}^{*}(\mathrm{~N} / \mathrm{k})^{2}\right)$, because the same model proposed by Lorek \& Sonnenschein was used [8]. In the Lorek model, the local territory notion does not exist. Each processor has global information of the whole territory and seeks neighbours in $\mathrm{N}$ fish positions, only obtaining good speedups with low and unrealistic granularity (speedup=16 with 10 fish per processor). 


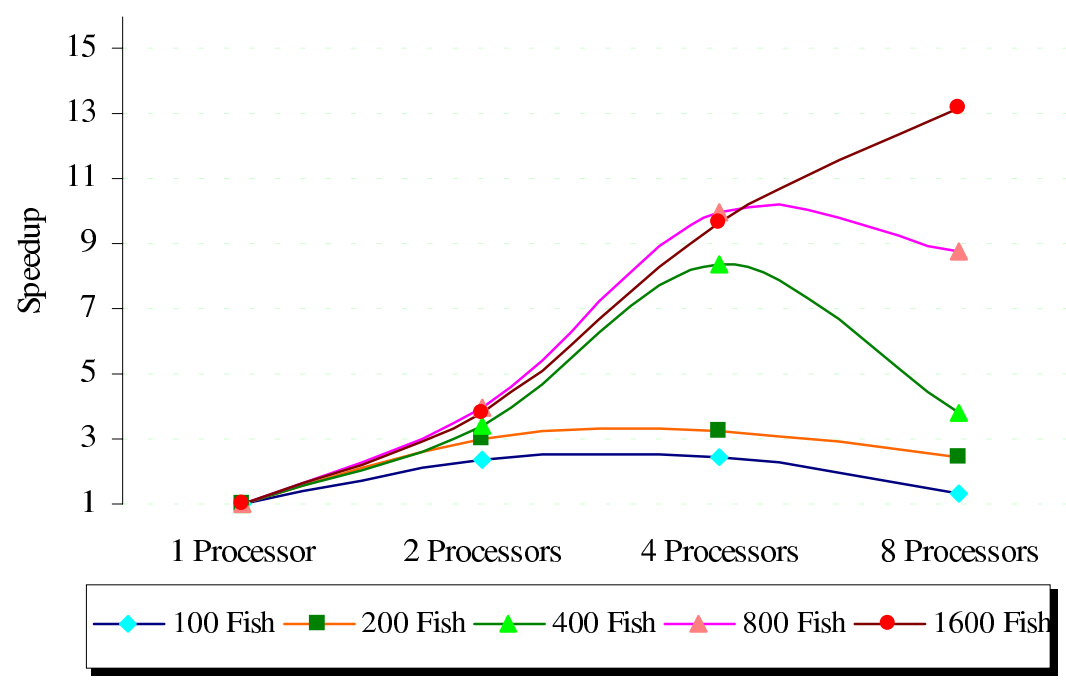

Fig. 7. Speedup

\section{Conclusions}

The ecological systems simulation is a field that requires considerable computing power for realistic models, and parallelism is a very useful tool in solving this type of problem. The present study shows very good results for IoM models and efficient solutions for such problems by using PDES. The operative prototypes were built using a PDES kernel developed at the UAB and are executed on a low-cost distributed architecture based on Linux.

The IoM Fish Schools model was developed using conservative and optimistic PDES algorithms. The results are better in the first type of protocols, due to the model characteristics. The main conclusions that can be extracted are:

- Model scalability is possible and acceptable. The division of the problem to be simulated into partitions (cubes) increases the speedup with respect to the existing parallel versions [8]. For a fixed size problem (number of fish) there is an optimal number of processor maximizing the speedup. Increasing the processors number should be accompanied of a problem size growing in order to keep a higher speedup.

- Performance is good with respect to large data animations, but there is a limit essentially imposed both by the communications model and the architecture. A reduction in this time would mean changing the communications model (for example using MPI) and modifying the network communications technology (Gigabit Ethernet).

Future work is guided towards: 
- The need to include within the study an increase in individual and processor numbers in order to verify model scalability.

- Simulation methods will have to include optimistic protocols such as the STW [11] that allows improvement in results by controlling the rollback chains.

- The IoM model must be improved, including biological characteristics to make it more realistic. This would involve, for example: speed coupling, behaviour patterns, inertial behaviours, dead angles, etc.

- The visualization of the simulation results must be improved in order to allow totally integrated animations with the simulation, by using (for example) OpenGL.

\section{References}

1. Booch, G., Rumbaugh. J., Jacobson, I. The unified modelling language: User's guide. Adisson-Wesley. (1999)

2. Cores, F. Switch Time Warp: Un método para el control del optimismo en el protocolo de simulacion distribuïda Time Warp. MsC Thesis (in Spanish). Universitat Autònoma de Barcelona. Spain. (2000)

3. Deelman, E., Coraco, T., Boleslaw, K. Parallel discrete event simulation of lime disease. Pacific Biocomputing Conference. (1996). 191-202.

4. ECOTOOLS: High level tools for modelling and simulation of individual-oriented ecological models. http://www.offis.uni-oldenburg.de/projekte/ecotools (1999)

5. Fishwick, P., Sanderson, J.G., Wolf, W. A multimodeling basis for across-trophic-level ecosystem modelling. Trans. SCS. Vol. 15. No. 2. (1998) 76-89.

6. Huth, A., Wissel, C. The simulation of movement of fish schools. Journal of Theoretical Biology 156. (1992) 365-385.

7. Kreft, J. Booth, G, Wimpenny, W. BacSim, a simulator for individual-based modelling of bacterial colony growth. Microbiology 144. (1998) 3275-3287.

8. Lorek, H, Sonnenschein, M. Using parallel computers to simulate individual oriented models: a case study. European Simulation Multiconference (ESM). (1995) 526-531.

9. Munt, P. Simulació distribuïda en PVM: Implementació dels algorismes TimeWarp i Switch Time Warp. Graduate Thesis (in Catalan). Universitat Autònoma de Barcelona. Spain. (1999)

10. Proctor, G., Winter, C. Information flocking, data visualisation in Virtual Worlds using emergent behaviours. Proc. Int. Conf. of Virtual Worlds Vol. 1434. Springer-Verlag. (1998) 168-176.

11. Suppi, R., Cores, F, Luque, E.. Improving optimistic PDES in PVM environments. Lecture Notes in Computer Science. Springer-Verlag 1908. (2000). 304-312.

12. Sloot, P. Kaandorp, J., Hoekstra, A., Overeinder, B. Distributed Cellular Automata: Large Scale Simulation of Natural Phenomena. Solutions to Parallel and Distributed Computing Problems: Lessons from Biological Sciences. ISBN: 0-471-35352-3 (2001), 1-46, 